\title{
Analysis by optical absorption and transmission electron microscopy of the strain inhomogeneities in InGaAs/InP strained layers
}

\author{
P. Roura \\ LCMM, Departament de Física Aplicada i Electrònica, Universitat de Barcelona, Facultat de Física, \\ Diagonal 645, E08028-Barcelona, Catalonia, Spain \\ S. A. Clark \\ Department of Physics and Astronomy, University of Wales, College of Cardiff, P.O. Box 913 \\ Cardiff CF2 3YB, Wales, United Kingdom \\ J. Bosch, F. Peira, A. Cornet, and J. R. Morante \\ LCMM, Departament de Fisica Aplicada i Electrònica, Universitat de Barcelona, Facultat de Física, \\ Diagonal 645, E08028-Barcelona, Catalonia, Spain
}

(Received 2 August 1994; accepted for publication 3 January 1995)

\begin{abstract}
Optical absorption spectra and transmission electron microscopy (TEM) observations on InGaAs/ InP layers under compressive strain are reported. From the band-gap energy dispersion, the magnitude of the strain inhomogeneities, $\sigma_{\epsilon}$, is quantified and its microscopic origin is analyzed in view of the layer microstructure. TEM observations reveal a dislocation network at the layer interface the density of which correlates with $\sigma_{\epsilon}$. It is concluded that local variations of dislocation density are responsible for the inhomogeneous strain field together with another mechanism that dominates when the dislocation density is very low. (c) 1995 American Institute of Physics.
\end{abstract}

\section{INTRODUCTION}

The most usual technique to obtain the mean strain and composition in strained epitaxial layers is double crystal $\mathrm{x}$-ray diffraction. ${ }^{1}$ When the stress is partially relaxed, it is necessary to measure the lattice parameter perpendicular and normal to the growing surface. Of course, the mean values of strain and composition give no information about the layer quality. As the composition inhomogeneities and the structural defects, such as dislocations, twins, or stacking faults broaden the diffraction peak, its width is taken as a measure of the layer quality. ${ }^{2,3}$ However, other factors such as sample curvature and layer thickness can also affect the peak broadening making a correct quantification difficult. Alternatively, observation by transmission electron microscopy (TEM) can give complementary information that is quantified in terms of defect density. ${ }^{3}$

In recent works, ${ }^{4,5}$ we have shown that the optical absorption $(\mathrm{OA})$ spectra near the band edge can deliver very detailed information. The analysis relies on the fact that the band-gap energy depends on the molar composition as well as the strain. Besides, the splitting of the heavy-hole ( $\mathrm{HH})$ and light-hole (LH) valence subbands increases with strain. So, from the band-gap energy and the valence-band splitting, the strain and the molar composition can be obtained with high accuracy. ${ }^{4}$ The main success of this technique is, however, the possibility of the quantification of the layer quality in terms of composition or strain inhomogeneities through the band-gap energy dispersion. It has been shown that, depending on the origin of microscopical inhomogeneities, the spectral shape changes. ${ }^{5}$ The $\mathrm{HH}$ energy dispersion $\left(\sigma_{\mathrm{HH}}\right)$ is smaller than that corresponding to the LH subband $\left(\sigma_{\mathrm{LH}}\right)$ if composition is not homogeneous. On the other hand, a ratio $\sigma_{\mathrm{HH}} / \sigma_{\mathrm{LH}}>1\left(\sigma_{\mathrm{HH}} \sigma_{\mathrm{LH}} \approx 2.8\right.$ for $\operatorname{In}_{x} \mathrm{Ga}_{1-x}$ As when $\left.x \approx 0.5\right)$ reveals an inhomogeneous strain field.
In this article we compare the dispersion in the strain value on InGaAs/InP layers under compressive strain determined by $\mathrm{OA}$ with the dislocation density measured by TEM. The good agreement between both techniques is the first experimental proof that the microscopical inhomogeneities can be quantified by $\mathrm{OA}$. It is concluded that, in partially relaxed layers, the strain inhomogeneities are related to the change in the dislocation density from point to point in the layer.

\section{EXPERIMENTAL RESULTS}

The samples analyzed in this work are $\mathrm{In}_{x} \mathrm{Ga}_{1-x}$ As epitaxial layers grown by molecular beam epitaxy on $\operatorname{InP}(100)$ with the growth conditions detailed in Ref. 1. Layer thickness ranges from 0.4 to $0.5 \mu \mathrm{m}$. The molar composition, $x$, and the mean strain, $\epsilon$ (Table I), are deduced from the fitting of the OA spectra. The OA measurements have been carried out with a Fourier transtorm IR spectrometer. A continuous flow helium cryostat allowed the samples to be held at $10 \mathrm{~K}$.

Typical OA spectra are shown in Fig. 1. The valenceband splitting due to strain is clearly resolved and, as the first band in the spectra is the more intense, ${ }^{4}$ it is concluded that the strain is compressive. To quantify properly the layer parameters, we have fitted the spectra with the theoretical shape given by Elliot's formula ${ }^{6}$

$$
\begin{gathered}
\alpha(h \nu)=A \frac{2 \pi \sqrt{\epsilon_{x}}}{h \nu} \sum \frac{2 \epsilon_{x}}{n^{3}} \delta\left(h \nu-E_{g}+\frac{\epsilon_{x}}{n^{2}}\right) \\
+\frac{H\left(h \nu-E_{g}\right)}{1-\exp \left(-2 \pi \sqrt{\epsilon_{x} / h \nu-E_{g}}\right)},
\end{gathered}
$$

where $\epsilon_{x}$ is the exciton binding energy and $H(x)$ is the Heavyside function. To account for the valence-band splitting, $\Delta E$, we consider that every subband has the same shape but a different band-gap energy, $E_{g}$. Finally, the microscopical inhomogeneities are introduced by convoluting every 
TABLE I. Layer characteristics (molar composition, $x$, mean strain, $\epsilon$ and strain dispersion, $\sigma_{\epsilon}$ ) obtained from optical absorption at $10 \mathrm{~K}$ and the relaxed strain, $\Delta \epsilon_{\mathrm{TEM}}$, deduced from TEM observations.

\begin{tabular}{ccccc}
\hline Sample & $x$ & $\epsilon\left(\times 10^{-3}\right)$ & $\sigma_{\epsilon}\left(\times 10^{-3}\right)$ & $\Delta \epsilon_{\mathrm{TEM}}\left(\times 10^{-3}\right)$ \\
\hline A & 54.9 & 1.0 & 0.28 & 0 \\
B & 55.7 & 1.6 & 0.26 & 0.33 \\
C & 57.5 & 2.5 & 0.35 & 0.33 \\
D & 58.0 & 2.6 & 0.59 & 0.87 \\
E & 59.4 & 2.3 & 0.50 & 0.59 \\
\hline
\end{tabular}

subband with a Gaussian distribution characterized by the standard deviations $\sigma_{\mathrm{HH}}$ and $\sigma_{\mathrm{LH}}$. The fitting parameters are detailed in Table II. The important result is the relative energy dispersion, $\sigma_{\mathrm{HH}} / \sigma_{\mathrm{LH}}$. In all samples we obtain a value between 2 and 2.8, which indicates that the microscopic inhomogeneities are essentially strain variations. Following the procedure given in Ref. 4, the layer parameters are summarized in Table I. As the band-gap energy changes linearly with strain, from the energy dispersion we can determine the strain dispersion $\left(\sigma_{\epsilon}\right)$ in the samples (Table I).

The TEM observations in all samples but layer A show a regular network of dislocations which are clearly seen in the plan view micrograph of Fig. 2. This is evidence that the stress is partially relaxed. Sample A is near the lattice-match composition, so it is coherent with the substrate and no dislocations or defects appear. On the other hand, sample $E$ is far from the lattice-match composition and a great number of defects other than perfect dislocations are developed (stacking faults and threading dislocations are clearly seen in Fig. 3).

\section{ANALYSIS}

The aim of this section is to understand the layer parameters deduced from a macroscopical technique as $\mathrm{OA}$ is, in view of the microscopic structure observed by TEM.

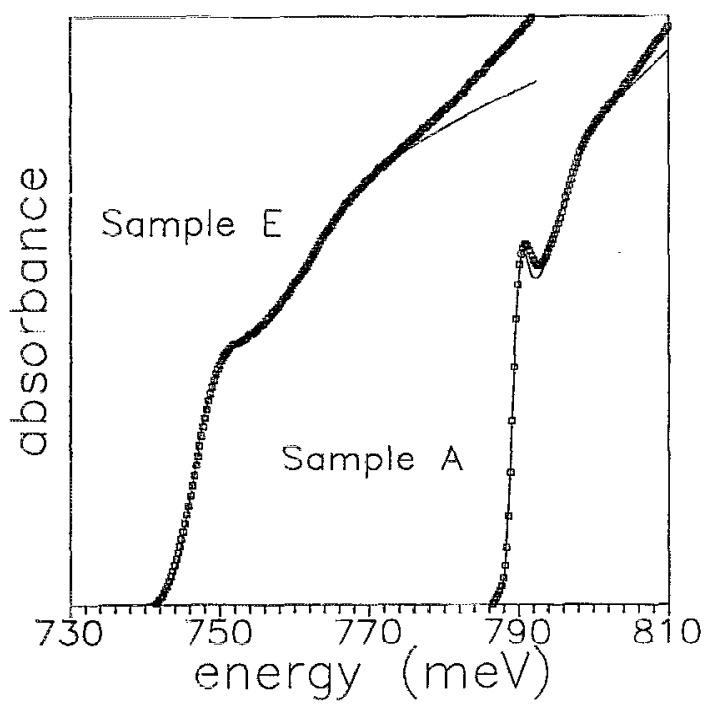

FIG. 1. Typical optical absorption spectra measured at $10 \mathrm{~K}$ (points) and their fitting (solid line).
TABLE II. Fitting parameters of the optical absorption spectra: exciton binding energy, $\epsilon_{x}$, band-gap energy, $E_{g}$, valence-band splitting, $\Delta E$, and the energy dispersions, $o_{\mathrm{HH}}$ and $\sigma_{\mathrm{LHF}}$. (Energies are given in meV.)

\begin{tabular}{clcccc}
\hline \hline Sample & $\epsilon_{x}$ & $E_{g}$ & $\Delta E$ & $\sigma_{\mathrm{LH}}$ & $\sigma_{\mathrm{HH}} / \sigma_{\mathrm{LH}}$ \\
\hline A & 0.9 & 790.8 & 6.9 & 0.9 & 3.0 \\
B & 1.46 & 784.5 & 11.7 & 0.9 & 2.8 \\
C & 0.75 & 768.3 & 17.7 & 1.2 & 2.8 \\
D & 0.9 & 763.3 & 18 & 2.3 & 2.8 \\
E & 0.9 & 748.4 & 16.4 & 2.4 & 2.0 \\
\hline \hline
\end{tabular}

In the ideal case where only a network of dislocations of angle $60^{\circ}$ are present, the amount of relaxed strain $\Delta \epsilon_{\mathrm{TEM}}$ can be calculated with the following formula: ${ }^{7}$

$$
\Delta \epsilon_{\mathrm{TEM}}=\frac{|b|}{2 d}
$$

where $d$ is the mean distance between dislocations and $b$ is the Burger's vector modulus. From the extinction conditions of the contrast we know that the dislocations are mainly of $60^{\circ}$ with the Burger's vector in the $\langle 110\rangle$ direction. Its modulus is proportional to the lattice constant a $(b=\langle 110\rangle a / 2)$ which is approximately that of the InP, $a_{\text {InP }}=5.87 \AA^{8} .^{8}$ So, $b=4.15 \AA$. The results are quoted in Table I. The subindex "TEM" indicates that this is the relaxed strain due to the network of $60^{\circ}$ dislocations. Provided that the actual strain relaxation is great enough, it could be obtained from the $\mathrm{OA}$ spectra. This procedure gives a significant value only in sample $E$, where the result is $\Delta \epsilon=1.7 \pm 0.2 \times 10^{-3}$. This is about three times greater than the strain relaxed by the dislocation network indicating that the other defects seen in Fig. 3 contribute to relaxation as well.

The central point of this study is the microscopic origin of the strain dispersion, $\sigma_{\epsilon}$, quantified by $O A$. In a first approximation we could think that the greater the relaxation, the greater the strain inhomogeneities. However this is not the case in view of the results because sample $A$ is not relaxed at all, yet it has a strain dispersion similar to $B$ and $C$. Further, sample $\mathrm{E}$ has the greatest strain relaxation. (deter-

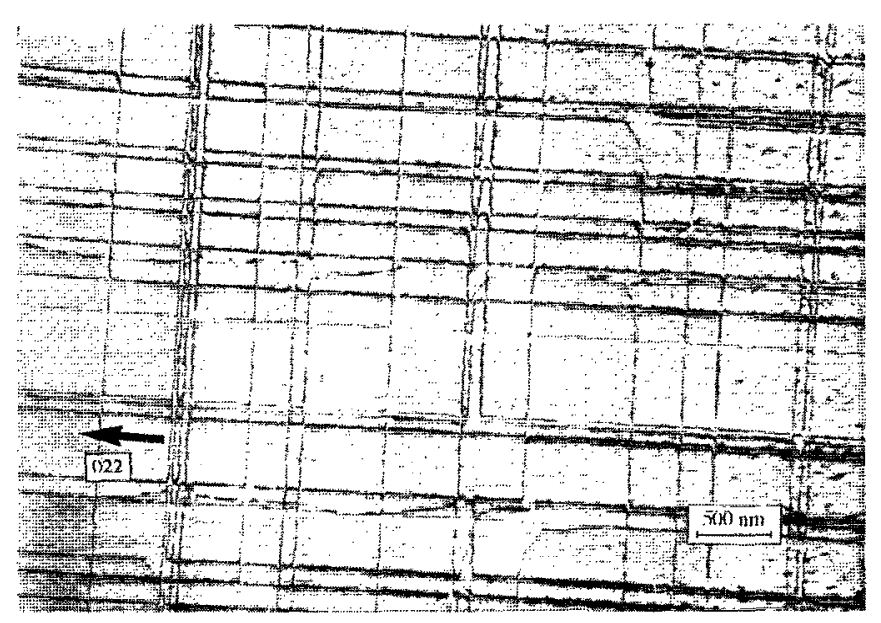

FIG. 2. Plan view of sample D. The dislocation network at the interface is clearly seen. 


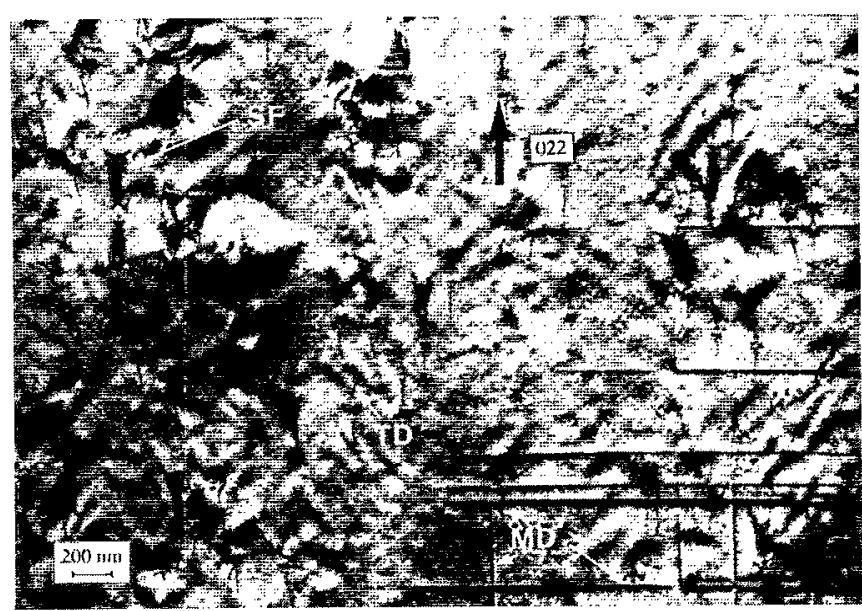

FIG. 3. Plan view of sample $\mathrm{E}$. Besides the dislocation network (MD) at the interface, a great density of threading dislocations (TD) and stacking faults (SF) is observed in the region where the substrate has been removed.

mined by $\mathrm{OA}$ ) whereas its $\sigma_{\epsilon}$ is smaller than that of sample $\mathrm{D}$ (Table I). In spite of these inconsistencies, there must be a relationship between the microscopic structure and the strain dispersion. We propose that the origin of $\sigma_{\epsilon}$ is the dislocation network. Locally, the strain can be.different from the mean value. However, as dislocations relax the strain, the departure from the mean value $\left(\sigma_{\epsilon}\right)$ cannot be greater than the relaxation, otherwise at some points the dislocations would increase the strain. This condition can be approximated as follows:

$$
\Delta \epsilon_{\mathrm{TEM}}>\sigma_{\epsilon} \text {. }
$$

In order to test our hypothesis, in Fig. 4 we have plotted $\sigma_{\epsilon}$ versus the inverse of the mean distance between dislocations, $d$. There is a clear correlation: $\sigma_{\epsilon}$ increases monotonically with $1 / d$. Further, in samples with greater dislocation densities, Eq. (3) is followed. This proves that the strain inhomogeneities are indeed due to the dislocation network. Other defects, such as stacking faults, have a minor effect on $\sigma_{\epsilon}$. We think that the strain inhomogeneities reflect the local variation of dislocation density (local plastic relaxation) and not the strain field surrounding each dislocation ${ }^{9}$ (local elastic relaxation). In this second situation, the inhomogeneities would be smoothed as dislocation density increases, which is not the case in view of Fig. 4. Besides, Fig. 4 tells us that, at low dislocation density, there must be another source of strain inhomogeneities. In particular, this is clearly seen in sample A, where no dislocations have been found. Of course, this unknown source of inhomogeneities can be present in the other samples as well. This can explain why sample $\mathrm{C}$ does not follow exactly Eq. (3).

At this point it is worthwhile to make a collateral discussion concerning the precision of $\mathrm{X}$-ray diffraction and $\mathrm{OA}$ in determining the molar composition. In both cases, this determination relies on the hypothesis that the lattice strain depends only on the molar composition and plastic relaxation. The fact that in sample A the strain dispersion is not due to composition variation (if this were the case, $\sigma_{\mathrm{HH}} \leqslant \sigma_{\mathrm{LH}}$ ) or plastic relaxation (absence of dislocations) means that the

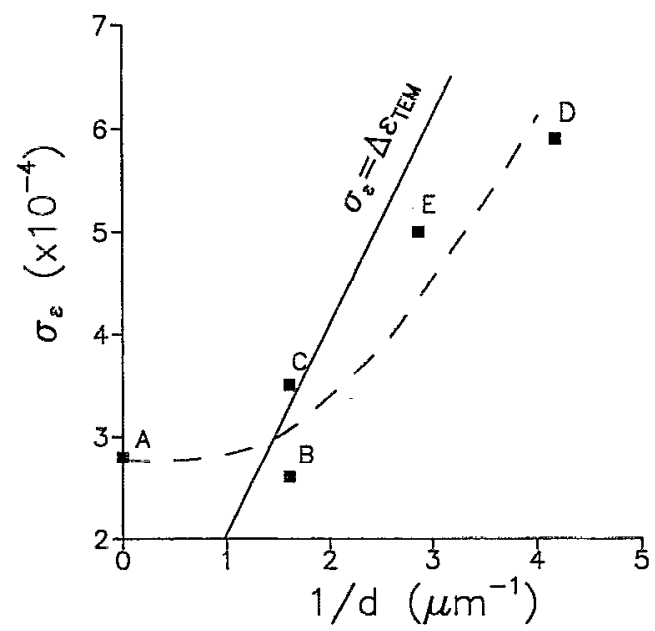

FIG. 4. Figure relating the strain dispersion, $\sigma_{\epsilon}$, quantified by $\mathrm{OA}$ and the mean distance between dislocations determined by TEM. If the source of strain inhomogeneities were the dislocations network all points would fall at the right-hand side of the straight line. The dashed curve shows the general trend.

strain may be due to another effect not accounted for in the analysis of $\mathrm{OA}$ and $\mathrm{X}$-ray measurements. This can explain why those techniques are more reliable in the measurement of strain than molar composition. ${ }^{4}$ Concerning the new source of strain we think it could be due to the existence of the oval defects usually encountered in this kind of layer.

\section{SUMMARY}

In summary, OA spectra and TEM observations have been made in InGaAs layers under compressive strain grown on InP. From the OA spectra it is concluded that the main microscopic inhomogeneities in the layers arise from an inhomogeneous strain field. Its origin has been analyzed by comparing the strain dispersion quantified by $\mathrm{OA}$ with the dislocation density determined by TEM. We conclude that there are two main sources of these strain inhomogeneities: the variations in the dislocation density from point to point in the layer and a mechanism not related to plastic relaxation that is predominant in layers near the lattice-matched composition.

\section{ACKNOWLEDGMENT}

This work was supported by the Spanish "Programa de Ciencia de Materiales" project under Contract No. MAT930564.

${ }^{1}$ S. A. Clark, J. E. McDonald, D. 1. Westwood, and R. H. Williams, J. Cryst. Growth 121, 743 (1992).

${ }^{2}$ I. C. Bassignana, C. J. Miner, and N. Puetz, J. Appl. Phys. 65, 4299 (1989).

${ }^{3}$ B. K. Tanner, J. Cryst. Growth 99, 1315 (1990).

${ }^{4}$ S. A. Clark, P. Roura, J. Bosch, A. Perez-Rodriguez, J. R. Morante, D. I. Westwood, and R. H. Williams, J. Appl. Phys. 77, 3393 (1995).

${ }^{5}$ P. Roura, J. Bosch, and J. R. Morante, Phys. Rev. B 46, 10453 (1992).

${ }^{6}$ R. J. Elliot, Phys. Rev. 108, 1384 (1957).

${ }^{7}$ P. M. J. Maree, J. C. Barbour, J. F. van der Veen, K. L. Kavanagh, C. W. T. Bulle-Lieuwma, and M. P. A. Viegers, J. Appl. Phys. 62, 4413 (1987). ${ }^{8}$ S. Adachi, J. Appl. Phys. 53, 8777 (1982).

${ }^{9}$ R. Bonet, Phys. Status Solidi B 108, 435 (1981). 